\title{
On the phase structure and thermodynamics of QCD
}

\author{
Tina Katharina Herbst, ${ }^{1,2}$ Jan M. Pawlowski, ${ }^{2,3}$ and Bernd-Jochen Schaefer ${ }^{1}$ \\ ${ }^{1}$ Institut für Physik, Karl-Franzens-Universität, A-8010 Graz, Austria \\ ${ }^{2}$ Institut für Theoretische Physik, University of Heidelberg, \\ Philosophenweg 16, D-69120 Heidelberg, Germany \\ ${ }^{3}$ ExtreMe Matter Institute EMMI, GSI, Planckstr. 1, D-64291 Darmstadt, Germany.
}

\begin{abstract}
We discuss the phase structure and thermodynamics of QCD by means of dynamical chiral effective models. Quark and meson fluctuations are included via the functional renormalization group. We study the influence of confinement in addition to the impact of fluctuations by comparing the results of the chiral models to their Polyakov-loop extended versions. Furthermore, we discuss the mass sensitivity of the phase structure and thermodynamics and find interesting modifications close to the chiral limit.
\end{abstract}

PACS numbers: 12.38.Aw, 11.10.-z, 11.30.Rd , 12.38.-t

\section{INTRODUCTION}

The properties of strongly-interacting matter at nonvanishing temperature and density are in the focus of many theoretical and experimental efforts. On the experimental side, future and running heavy-ion experiments at various facilities such as GSI, JINR, CERN and BNL aim at probing the phase structure of quantum chromodynamics (QCD), especially in the regime where potentially a critical endpoint (CEP) is present.

On the theoretical side, functional continuum methods are well suited for a combined study of the confinementdeconfinement and chiral phase structure of QCD at finite density. In recent years, functional renormalization group (FRG) studies and Dyson-Schwinger (DSE) studies have provided valuable insights into the phase structure of strongly interacting matter. These studies were performed both within first principle QCD as well as within low-energy effective models, see [1-7]. For QCDrelated reviews see $[4,8-16]$ (FRG) and [17-22] (DSE).

At vanishing density, lattice simulations provide additional insights from first principle QCD that are complementary to the continuum studies. This also helps to improve the systematic error analysis of the respective approaches. At nonvanishing chemical potential, however, lattice simulations are hampered by the sign problem, see e.g. [23, 24]. Impressive progress in overcoming this problem has been made [25-31], but to date lattice QCD is still restricted to small chemical potential.

Low-energy effective models, such as the Nambu-JonaLasinio (NJL) and quark-meson (QM) models, are known to describe the chiral dynamics of QCD rather well; for a recent review see e.g. [32]. Polyakov-loop extended versions thereof (PNJL, PQM) [33-35], have advanced our understanding of the confinement-deconfinement aspects of the QCD phase structure, e.g. [2, 3, 34-45]. It has been known for a long time, e.g. [9, 11, 12, 14, 15], that low-energy effective models can be systematically related to full QCD within the FRG approach. For Polyakovloop extended models this follows from the Landau gauge approach in $[1,4,46]$, and for the Polyakov gauge, see $[47,48]$. This setting has been discussed in detail for the PQM model in $[3,4,49]$; see also our discussion in Sec. II. While chiral symmetry and its dynamical breaking are well described within these models, confinement is only included in a statistical manner. Moreover, the glue potential of full QCD, encoding the gauge dynamics in the presence of matter fields, is replaced by a Polyakovloop potential. This potential is fixed to lattice data of the pure Yang-Mills system at vanishing chemical potential. In such an approach, the coupling of the matter sector to the gauge sector is lost, e.g. [3, 35]. This is discussed in detail below, where we show how the matter back-coupling can effectively be taken into account.

In conclusion, the FRG approach to the low-energy dynamics of QCD is a promising setup for investigating the QCD phase structure and thermodynamics. A study along these lines has already been put forward in [3, 49]. In the present work we extend this approach by a more refined inclusion of the matter and glue dynamics as well as by studying the mass sensitivity of various observables.

This paper is structured as follows: in Sec. II we introduce the Polyakov-quark-meson model as a low-energy truncation of full two-flavor QCD. Furthermore, the inclusion of the matter back-coupling to the gauge sector is discussed. Section II A summarizes the renormalization group approach and the resulting flow equation for our model. In the following Sec. III we compare the phase structure of the PQM model to that of the QM model and discuss the mass sensitivity of the phase transitions. In particular, we find an intriguing splitting of the chiral phase transition at low Goldstone-boson masses, which is only observed when fluctuations are taken into account. Some thermodynamic observables and their mass dependence are studied in Sec. IV. Concluding remarks and an outlook are given in Sec. V and some details of our numerical implementation can be found in the Appendix.

\section{FROM QCD TO POLYAKOV-LOOP EXTENDED CHIRAL MODELS}

We have already emphasized in the introduction that low-energy effective models can be related to full QCD in 


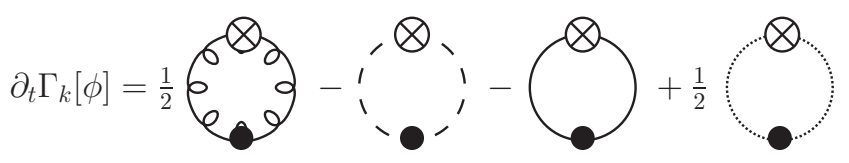

FIG. 1: Partially hadronized version of the FRG flow for QCD. The loops denote the gluon, ghost, quark and meson contributions, respectively. The crosses mark the RG regulator term.

a systematic fashion. In [1, 3, 4, 47-49] this is discussed for the embedding of Polyakov loop extended models: The FRG provides a setting which allows to approach the low temperature regime of QCD from large momentum scales by successively integrating out momentum shells. This entails that one starts at asymptotically large momenta with perturbative QCD with one coupling parameter, the strong coupling $\alpha_{s}\left(p^{2}\right)$. The other parameters are the entries in the quark mass matrix. In the present two-flavor study the masses of up and down quark are small and the mass matrix is diagonal in flavor space.

When lowering the momentum scale one systematically includes quark and gluon fluctuations into the theory, finally approaching the hadronic phase; see [50] for one flavor QCD and $[1,4]$ for two-flavor QCD. Close to the phase boundary between the quark-gluon plasma phase and the hadronic phase and at not too large chemical potential, mesonic degrees of freedom, in particular the pion and sigma fluctuations, become important. The related quark bilinears, $\bar{q} q$ and $\bar{q} \gamma_{5} \vec{\tau} q$, carry the same quantum numbers, i.e. they have a considerable overlap with the full meson operators. The matter sector of the QCD effective action can be conveniently written in powers of these bilinears and related kinetic terms. The strength and the momentum dependence is systematically computed by means of the flow equation for QCD, depicted in Fig. 1. The propagators in Fig. 1 are the fully dressed field dependent propagators and the flow equation describes the fully coupled QCD glue-matter system. In particular, there are contributions of the matter sector also in the diagrams for the gauge sector, i.e., the vacuum polarization diagram in the gluon propagator, e.g. Fig. 2. The first two loops in Fig. 1 constitute the glue potential of QCD. Note that this is not the Yang-Mills potential, as the gluon and ghost propagator are those of QCD and scale differently with momenta. In turn, dropping the matter back-coupling these two loops correspond to the flow of the glue potential of pure Yang-Mills theory [51-53], and for more details see [4, 54]. The remaining two loops represent the flow equation of the dynamical quark-meson model, e.g. [55, 56].

Following these arguments, we employ the Polyakovquark-meson (PQM) model $[3,35]$ as an effective realization for low-energy QCD. As mentioned in the introduction, the PQM model is an extension of the quark-meson (QM) model by Polyakov-loop variables which allows to effectively describe chiral and certain aspects of confinement in QCD. The Euclidean Lagrangian of this model

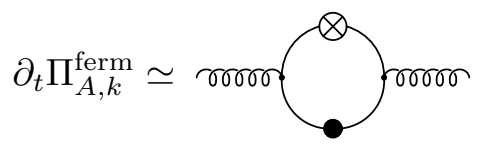

FIG. 2: Quark polarization contribution to the gluon propagator representing a contribution to the matter back-coupling.

for $N_{f}=2$ flavors and $N_{c}=3$ colors including a uniform quark chemical potential $\mu$, reads

$$
\begin{aligned}
\mathcal{L}_{\mathrm{PQM}}= & \bar{q}\left(\not D+h\left(\sigma+i \gamma_{5} \vec{\tau} \vec{\pi}\right)+\mu \gamma_{0}\right) q+\frac{1}{2}\left(\partial_{\mu} \sigma\right)^{2} \\
& +\frac{1}{2}\left(\partial_{\mu} \vec{\pi}\right)^{2}+U(\sigma, \vec{\pi})+\mathcal{U}\left(\Phi, \bar{\Phi} ; T_{0}\right),
\end{aligned}
$$

where $\phi=(\sigma, \vec{\pi})$ denotes the $O(4)$-symmetric representation of the meson fields. The scalar sigma meson and the three pseudoscalar pions are coupled via a flavor-blind Yukawa coupling $h$ to the quark fields, $q$ and $\bar{q}$. The covariant derivative $\not D(\Phi)=\gamma_{\mu} \partial_{\mu}-i g \gamma_{0} A_{0}(\Phi)$ couples the Polyakov loop, defined as

$$
\Phi(\vec{x})=\frac{1}{N_{c}}\left\langle\operatorname{tr} \mathcal{P} \exp \left(i g \int_{0}^{\beta} d \tau A_{0}(\vec{x}, \tau)\right)\right\rangle,
$$

to the quark fields. In this work we describe the theory solely in terms of the Polyakov loop $\Phi$ and suppress the $A_{0}$ dependence. Differences between these two formulations will be discussed elsewhere. Moreover, we add a purely mesonic potential $U(\sigma, \vec{\pi})$ that allows for explicit as well as spontaneous breaking of chiral symmetry

$$
U(\sigma, \vec{\pi})=\frac{\lambda}{4}\left(\sigma^{2}+\vec{\pi}^{2}-v^{2}\right)^{2}-c \sigma .
$$

The explicit breaking of chiral symmetry is realized by a term linear in the sigma field. The pure quark-meson (QM) model is obtained from the PQM model by setting $A_{0}=0$, i.e. $\Phi, \bar{\Phi}=1$ and omitting the glue potential $\mathcal{U}\left(\Phi, \bar{\Phi} ; T_{0}\right)$.

In Polyakov-loop extended chiral effective models, the glue potential of full QCD is usually approximated by an effective Polyakov-loop potential $\mathcal{U}\left(\Phi, \bar{\Phi} ; T_{0}\right)$, which is constructed by means of a Landau-Ginzburg-like ansatz. The arising coefficients are fitted to lattice results for the pure-glue system, see, e.g. Eq. (4) below. The explicitly shown parameter $T_{0}$ determines the scale of the deconfinement phase transition.

In the following we apply a simple polynomial ansatz for the glue potential as introduced in [40],

$$
\frac{\mathcal{U}}{T^{4}}=-\frac{b_{2}\left(T ; T_{0}\right)}{2} \Phi \bar{\Phi}-\frac{b_{3}}{6}\left(\Phi^{3}+\bar{\Phi}^{3}\right)+\frac{b_{4}}{4}(\Phi \bar{\Phi})^{2},
$$

with temperature-dependent coefficient

$$
b_{2}\left(T ; T_{0}\right)=a_{0}+a_{1}\left(\frac{T_{0}}{T}\right)+a_{2}\left(\frac{T_{0}}{T}\right)^{2}+a_{3}\left(\frac{T_{0}}{T}\right)^{3} .
$$


The parameters of Eqs. (4) and (5) have been determined in [40] by a fit to pure Yang-Mills lattice results to be

$$
a_{0}=6.75, a_{1}=-1.95, a_{2}=2.625, a_{3}=-7.44
$$

and

$$
b_{3}=0.75, \quad b_{4}=7.5 .
$$

In this construction, however, the backreaction of the matter sector is completely neglected. This deficiency can be overcome by replacing the constant parameter $T_{0}$ by a flavor number, $N_{f}$, and quark chemical potential, $\mu$, dependent function: $T_{0} \rightarrow T_{0}\left(N_{f}, T, \mu\right)$. For the $N_{f}$ and $\mu$ dependence of $T_{0}$ we follow the arguments in [3,35], to wit

$$
T_{0}\left(N_{f}, T, \mu\right)=T_{\tau} e^{-1 /\left(\alpha_{0} b\left(N_{f}, T, \mu\right)\right)},
$$

with the $\tau$-scale $T_{\tau}$ and

$$
b\left(N_{f}, T, \mu\right)=\frac{11 N_{c}-2 N_{f}}{6 \pi}-b_{\mu} \frac{\mu^{2}}{\left(\hat{\gamma} T_{\tau}\right)^{2}} \theta_{b}(T, \mu) .
$$

For details on our choice of parameters see [3]. The sensitivity of the phase structure to the parameter $\hat{\gamma}$ is discussed in Appendix B. There, we have varied this parameter in the range $0.5 \leq \hat{\gamma} \leq \infty$. The lowest value, $\hat{\gamma}=0.5$, corresponds to an unphysical $T_{0}(\mu)$, which tends to zero already at small $\mu \approx 300 \mathrm{MeV}$. Hence we have chosen $\hat{\gamma}=0.85$ for all figures in the main text, which is also suggested by a comparison to the HDL approximation (see also the discussion in [35]). With this value, the chiral and deconfinement transitions lie close to each other throughout the whole phase diagram (see Sec. III below).

The $\mu$-dependent correction in Eq. (9) is proportional to a difference of (baryonic) Fermi-Dirac distributions

$$
\theta_{b}(T, \mu)=n_{b}(T, \mu)+n_{b}(T,-\mu)-2 n_{b}(T, 0),
$$

with

$$
n_{b}(T, \mu)=\frac{1}{1+e^{3\left(m_{q}-\mu\right) / T}},
$$

and $m_{q}=h \sigma_{\mathrm{vac}}$ and $\sigma_{\mathrm{vac}}=f_{\pi}=93 \mathrm{MeV}$. The physical importance of this expression is seen in the limit of vanishing temperature. Then $\theta_{b}$ reduces to the step function $\theta\left(\mu-m_{q}\right)$, as it should in order to account for the Silver-Blaze property of QCD: at vanishing temperature, chemical potential effects are expected to only set in when $\mu \geq m_{q}$. Note that in the present approximation the binding energy of the nucleons is neglected. As the temperature increases, the sharp behavior of the step function is smoothed out.

The origin of $b\left(N_{f}, T, \mu\right)$ is the flow of the fermionic part of the vacuum polarization, see Fig. 2, or more precisely

$$
\left.\partial_{\vec{p}^{2}} \partial_{t} \Pi_{A, k}^{\mathrm{ferm}}\right|_{p^{2}=0}
$$

in the presence of the Polyakov loop. This term carries the fermionic part of the QCD $\beta$ function. Its $\mu$ dependence stems from the Polyakov-loop enhanced quark/antiquark occupation numbers,

$$
\begin{aligned}
& N_{q}(T, \mu ; \Phi, \bar{\Phi}) \\
& \quad=\frac{1+2 \bar{\Phi} e^{\left(E_{q}-\mu\right) / T}+\Phi e^{2\left(E_{q}-\mu\right) / T}}{1+3 \bar{\Phi} e^{\left(E_{q}-\mu\right) / T}+3 \Phi e^{2\left(E_{q}-\mu\right) / T}+e^{3\left(E_{q}-\mu\right) / T}} .
\end{aligned}
$$

with $E_{q}=\left(k^{2}+m_{q}^{2}\right)^{1 / 2}$, which has been used in $[1,4]$. Note that the occupation number Eq. (13) appears in any quark loop with a Polyakov loop or $A_{0}$ background. In fact, the flow of the fermionic part of the free energy both in QCD, [1, 4] and in the PQM-model, [2, 3] as well as Eq. (15), are proportional to Eq. (13). The latter flow is also central for the present work, e.g. Eq. (15) below.

Here we use Eq. (13) to estimate the $\mu$ dependence of the QCD $\beta$ function at vanishing cutoff scale, $k=0$ : For large temperatures $T \gtrsim T_{c}(\mu)$ and hence deconfining Polyakov loops, $\Phi, \bar{\Phi} \rightarrow 1$, the thermal distribution factor in Eq. (13) reduces to the standard Fermi-Dirac distribution for quarks, e.g. [50]. In this large temperature limit we also infer that $N_{q} \rightarrow 1 / 2$. In turn, for temperatures $T \lesssim T_{c}(\mu)$ and confining Polyakov loops with $\Phi, \bar{\Phi} \rightarrow 0$, and $\sigma=\sigma_{\text {vac }}$, we arrive at $n_{b}(T, \mu)$ in Eq. (11). Due to the $\Phi, \bar{\Phi}$ independence of the large temperature limit we simply use confining Polyakov loops $\Phi, \bar{\Phi}=0$ for all temperatures. This leads to our final expression Eq. (10).

In summary, the nontrivial factor $\theta_{b}$ in the definition $T_{0}\left(N_{f}, T, \mu\right)$ improves the original choice of $b\left(N_{f}, \mu\right)$ in [35] with respect to the thermodynamical properties by taking into account the dynamical change of the quark contributions to the glue sector for finite temperature and chemical potential.

\section{A. Flow equation for the PQM model}

For a realistic description of phase transitions one has to include thermal and quantum fluctuations. In the present work this is done within the functional renormalization group (Wetterich) equation [57]

$$
\partial_{t} \Gamma_{k}[\chi]=\frac{1}{2} \operatorname{STr}\left[\left(\Gamma_{k}^{(2)}[\chi]+R_{k}\right)^{-1} \partial_{t} R_{k}\right] .
$$

In this equation $t=\log (k / \Lambda)$ denotes the RG time, $\chi$ represents a generic field variable and $R_{k}$ is the RG regulator that implements the idea of integration over momentum shells. The supertrace STr, involves a trace over internal (color, flavor and Dirac) spaces as well as a momentum integration. Furthermore, it accounts for the correct signs of the fermionic and bosonic contributions.

After integrating out the gluonic degrees of freedom, the flow of the free energy contains solely the last two loops in Fig. 1, while the first two lead to the glue potential $\Omega_{\text {glue }}(\Phi, \bar{\Phi})$. Moreover, this procedure results in modifications of the matter sector due to the coupling to 
the Polyakov loops. This is accounted for by our initial conditions for the quark-meson sector. The QCD free energy is thus given by

$$
\begin{aligned}
\Omega_{\mathrm{QCD}}(\sigma, \vec{\pi}, \Phi, \bar{\Phi}) & =\Omega_{\text {glue }}(\Phi, \bar{\Phi})+\Omega_{\text {matter }, \Lambda}(\sigma, \vec{\pi}, \Phi, \bar{\Phi}) \\
& +\int_{\Lambda}^{0} d k \partial_{k} \Omega_{\text {matter }, k}(\sigma, \vec{\pi}, \Phi, \bar{\Phi}) \cdot(15)
\end{aligned}
$$

Since the gluons have been integrated out, their dynamics is stored in the Polyakov-loop glue potential $\Omega_{\text {glue }}$. Moreover, the full QCD free energy is necessarily independent of the cutoff, i.e., $\partial_{\Lambda} \Omega_{\mathrm{QCD}}=0$. Hence, $\Lambda$-dependent terms appear in $\Omega_{\text {matter }, \Lambda}$. These can be determined from the flow at $\Lambda$, e.g. $[8,9,11-13,15]$. From a phenomenological point of view these contributions correspond to the high-energy part of the vacuum fluctuations [58]. Finally, the flow equation for the matter sector of two-flavor QCD in a Polyakov loop or $A_{0}$-background reads [1-3]

$$
\begin{gathered}
\partial_{t} \Omega_{k}=\frac{k^{5}}{12 \pi^{2}}\left\{\frac{1}{E_{\sigma}} \operatorname{coth}\left(\frac{E_{\sigma}}{2 T}\right)+\frac{3}{E_{\pi}} \operatorname{coth}\left(\frac{E_{\pi}}{2 T}\right)\right. \\
\left.-\frac{4 N_{c} N_{f}}{E_{q}}\left[1-N_{q}(T, \mu ; \Phi, \bar{\Phi})-N_{q}(T,-\mu ; \bar{\Phi}, \Phi)\right]\right\},
\end{gathered}
$$

with the Polyakov loop enhanced particle numbers $N_{q}$ defined in Eq. (13). The quasiparticle energies are given by $E_{i}=\sqrt{k^{2}+m_{i}^{2}}, i=q, \pi, \sigma$, and the masses are defined as

$$
\begin{aligned}
& m_{q}^{2}=h^{2} \phi^{2}, \\
& m_{\pi}^{2}=2 \Omega_{k}^{\prime}, \\
& m_{\sigma}^{2}=2 \Omega_{k}^{\prime}+4 \phi^{2} \Omega_{k}^{\prime \prime} .
\end{aligned}
$$

In the above expressions, a prime at the potential denotes the derivative with respect to $\phi^{2}$.

The order parameters $\chi_{0}=\left(\sigma_{0}, \Phi_{0}, \bar{\Phi}_{0}\right)$ for given temperature and chemical potential are determined by the solution of the corresponding equation of motion (EoM)

$$
\left.\frac{\partial \Omega_{k \rightarrow 0}}{\partial \sigma}\right|_{\chi_{0}}=\left.\frac{\partial \Omega_{k \rightarrow 0}}{\partial \Phi}\right|_{\chi_{0}}=\left.\frac{\partial \Omega_{k \rightarrow 0}}{\partial \bar{\Phi}}\right|_{\chi_{0}}=0 .
$$

For the numerical solution of the coupled Eqs. (18), we utilize a stochastic technique which is outlined in Appendix A. In comparison to standard multidimensional root-finding algorithms, such as Newton's method, we obtained with this technique a much higher numerical accuracy within reasonable CPU time, which is required in particular for the evaluation of thermodynamic quantities.

\section{B. Initial condition at vanishing and finite temperatures and density}

In order to solve the flow equation (16) numerically, the parameters $\lambda, v^{2}$ and $c$ of the meson potential $U(\sigma, \vec{\pi})$ in Eq. (3) as well as the Yukawa coupling $h$ have to be specified at the UV scale. However, the parameters are not independent and are related to vacuum low-energy observables in the IR. For example, the explicit chiral symmetry breaking parameter $c$ relates the pion decay constant and the pion mass via $m_{\pi}^{2} f_{\pi}=c$ and can thus be fixed by these observables. The remaining three parameters at a given UV cutoff are chosen such that specific values of low-energy observables $\left(f_{\pi}, m_{\sigma}, m_{q}\right)$ are reproduced in the IR. In particular, the physical mass point is characterized by the values $f_{\pi}=\sigma_{\mathrm{vac}}=93 \mathrm{MeV}$, $m_{\pi}=138 \mathrm{MeV}$ and $m_{q}=h \sigma_{\mathrm{vac}}=297 \mathrm{MeV}$. The only insecure and experimentally not precisely known quantity is the sigma mass which affects the phase structure, see, e.g. [44]. To compare with previous works we choose for the sigma meson mass $m_{\sigma}=540 \mathrm{MeV}$, cf. [59].

To achieve these values in the infrared we have fixed the following initial values at the UV scale $\Lambda=950 \mathrm{MeV}$ :

$$
\begin{aligned}
\lambda & =1.3, \\
v^{2} & =-4.36 \cdot 10^{6}(\mathrm{MeV})^{2}, \\
c & =1.77 \cdot 10^{6}(\mathrm{MeV})^{3}, \\
h & =3.2 .
\end{aligned}
$$

Implicitly, Eq. (19) also takes into account the gluonic fluctuations: below the UV scale $\Lambda$ they effectively decouple, but their contributions above the UV scale lead to the initial conditions Eq. (19) and the Polyakov-loop potential. Moreover, for sufficiently large UV scale $\Lambda$, thermal and chemical potential modifications are negligible as they are suppressed exponentially with $-\Lambda / T$ for thermal fluctuations and polynomially with $\mu / \Lambda$ for density-related fluctuations. For large temperatures and/or chemical potential, however, the initial conditions in Eq. (19) receive corrections.

In general, thermal and quantum fluctuations with momenta $k \leq \Lambda$ have to be taken into account in a temperature and chemical potential dependence of Eq. (19). This can be either done by solving the related QCD flow for $k \geq \Lambda$ or by projecting this flow on the matter sector.

In our previous work [3] we have improved the thermodynamic observables by including the integrated UV flow in the initial condition of the free energy,

$$
\Omega_{\mathrm{matter}, \Lambda}[\sigma, \vec{\pi}, \Phi, \bar{\Phi}]=U(\sigma, \vec{\pi})+\Omega_{\Lambda}^{\infty}[\sigma, \vec{\pi}, \Phi, \bar{\Phi}],
$$

evaluated on the EoM. Here, $\Omega_{\Lambda}^{\infty}$ corresponds to the integration over the flow for the interacting Polyakov-loop system for scales $k \geq \Lambda$ and fixed parameters. This ensures the $\Lambda$ independence of system at $k=0$, i.e. RGinvariance. A more detailed discussion this procedure can be found e.g. in Refs. [2, 3, 58].

In the present work we extend this procedure to the full initial condition of the effective action. This is implicitly based on the results for the coupling flows in QCD with partial or full dynamical hadronisation $[1,4,60]$ : the flow of the QCD coupling parameters directly related to the parameters Eq. (19) is small, and hence the computation 
of the thermal and chemical potential modifications for $k \geq \Lambda$ on the basis of the $T=0$ values at $\Lambda$ is already a good estimate of the full modification in QCD. More details on this will be presented elsewhere.

\section{MASS SENSITIVITY OF THE PHASE STRUCTURE}

For the subsequent analysis of the mass sensitivity of the phase structure and thermodynamics, we will only vary the explicit chiral symmetry breaking parameter $c$, which determines the Goldstone-boson mass and keep all other parameters fixed.

From the order parameters we can deduce the chiral and deconfinement phase transition lines $T_{\chi}(\mu), T_{d}(\mu)$, respectively, in the $(T, \mu)$-phase diagram. At physical pion masses and small chemical potential, both transitions are crossovers, entailing that there exists no unique definition of the transition temperature. In the following we use the inflection point of the corresponding order parameter to define a (pseudo-)critical temperature. In addition, we compare this transition point to the one defined by one half of the normalized order parameter

$$
\frac{\sigma\left(T_{\chi}, \mu_{\chi}\right)}{\sigma(0,0)}=\frac{1}{2} .
$$

In order to highlight not only the influence of fluctuations, but also the direct impact of the Polyakov loop on the phase structure, we compare results obtained with the PQM model with those of the pure QM model. Furthermore, all PQM results presented below include the matter back-coupling $T_{0}\left(N_{f}, T, \mu\right)$, as introduced in Eq. (8).

\section{A. Physical mass point}

We begin with a discussion of the phase structure for physical values of the low-energy observables. The corresponding $(T, \mu)$-phase diagram for the QM model is shown in the left panel of Fig. 3. At low quark chemical potential we find a chiral crossover around $T \approx 160 \mathrm{MeV}$ which turns into a first-order phase transition at large chemical potential in a critical endpoint (CEP).

In comparison to standard mean-field results, where meson fluctuations are ignored, we observe that the location of the CEP is shifted towards lower temperatures when fluctuations are taken into account, see, e.g. also, [56]. Interestingly, a similar behavior is found if the standard mean-field approximation is improved by considering the renormalized QM models. The renormalization of these models amounts to including vacuum fluctuations of the quark loop [61,62]. Already these fluctuations push the location of the CEP towards higher chemical potential and smaller temperatures and exclude the existence of a CEP at low $\mu / T$ ratios. A similar conclusion can be drawn for renormalized (P)QM models with three quark flavors [63]. Note that these vacuum fluctuations are always included in the FRG treatment.

In addition, in Fig. 3 we compare the transition line obtained by the inflection point of the chiral order parameter (short-dashed line) with the one obtained via Eq. (21) (long-dashed). At low chemical potential both curves agree well, but start to deviate at larger chemical potential. The curve defined by the inflection point runs into the $\mathrm{CEP}$ and the transition turns into a firstorder one for smaller temperatures. A for RG calculations typical back-bending of the transition curve at low temperatures towards smaller chemical potential is observed. This behavior is not seen for the other definition of the chiral transition line, which does not reach the endpoint. In contrast, the curve bends outwards at lower temperatures and hits the $\mu$ axis at $\mu \approx 322 \mathrm{MeV}$.

In the right panel of Fig. 3 we include the Polyakov loop and show the PQM phase structure for the same IR values. At vanishing and low chemical potential, the chiral and deconfinement transitions are both crossovers (see [64] for the corresponding lattice results) and again both definitions of the chiral transition are shown. For the chiral transition, a similar behavior as in the pure QM model is found.

The Polyakov-loop related transitions, which we refer to as "deconfinement" transition lines, are denoted by the two black (dot-dashed and dot-dot-dashed) lines, defined by the inflection points of the Polyakov loop and its conjugate. The dark band in Fig. 3 denotes the width of $d \Phi / d T$ at $80 \%$ of its maximum height and measures the strength of the phase transition. At vanishing chemical potential the chiral and deconfinement transitions lie close to each other and continue to coincide for increasing chemical potential. However, if the matter backcoupling is neglected, a splitting of the two transitions at finite chemical potential has been observed in previous mean-field [35, 63] and RG calculations [3]. In particular, the deconfinement transition line becomes almost $\mu$ independent, i.e., $T_{d}(\mu) \approx T_{d}(\mu=0)$ without the matter backreaction. This effect supported previous speculations about a possible quarkyonic matter region in the QCD phase diagram, where chiral symmetry is restored while deconfinement persists [65]. In the present twoflavor study including fluctuations as well as the matter backreaction we, however, do not observe such a region anymore. A similar scenario has also been found in recent nonperturbative Dyson-Schwinger studies for twoand $(2+1)$-flavors, cf. [7,66] and references therein. The reader is referred to Appendix B for a discussion of the impact of the parameter $\hat{\gamma}$ on this conclusion.

Towards the critical endpoint, a narrowing of the dark band is seen that indicates a sharpening of the transitions. For temperatures below the CEP, the chiral transition is of first order. Also in the PQM model, the CEP is located at low temperatures, $\left(\mu_{\mathrm{CEP}}, T_{\mathrm{CEP}}\right) \approx$ $(290,20) \mathrm{MeV}$.

At this point, a word of caution concerning the highchemical potential region should be added. In this re- 

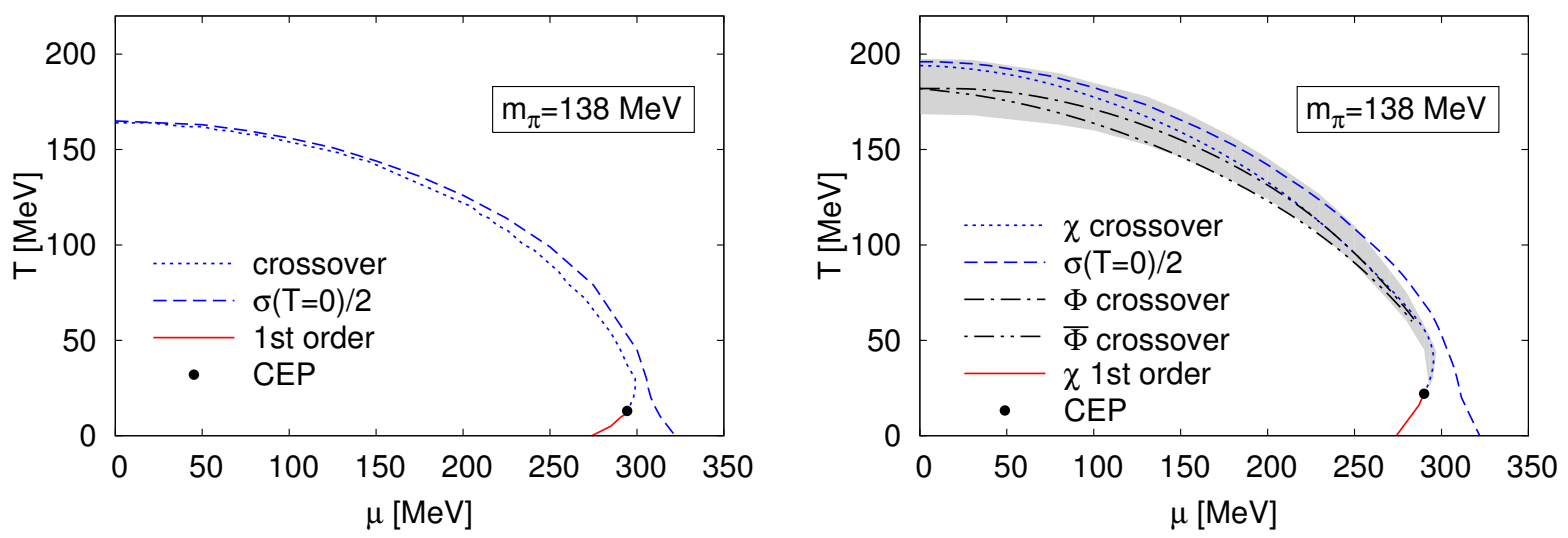

FIG. 3: Phase structure at physical pion masses for the QM (left) and PQM (right) models. Depicted is the chiral (blue, longand short-dashed) transition line. In the PQM model the Polyakov-loop transitions (black, dot-dashed and dot-dot-dashed lines) are also shown.

gion important baryonic degrees of freedom and diquark fluctuations are usually ignored in the literature. Due to their complexity this is also done in the present work. Recent estimates of the influence of baryons on the phase structure for two colors can be found, e.g., in [67-70]. Due to these omissions the phase structure at high chemical potential becomes questionable. However, the region that is to date accessible to lattice calculations, $\mu / T \leq 1$, is well within the region of applicability of the present study. Hence, we can safely conclude that for low chemical potential the existence of a critical endpoint can be excluded, see, e.g., also [71].

\section{B. Chiral limit}

In the following, we investigate the mass sensitivity of the phase structure. As an extreme case we consider the chiral limit, where no explicit chiral symmetry breaking terms are present and the Goldstone bosons, the pions, are massless. In this limit the chiral transition at vanishing chemical potential is a sharp transition of second order lying in the $O(4)$-universality class, cf. [72]. The resulting chiral phase structure is shown in Fig. 4. Compared to physical masses the critical temperature of the chiral transition decreases by approximately $20 \mathrm{MeV}$ due to the decreasing constituent quark masses. Since there is no ambiguity in the definition of the transition temperature in this limit, we only show one transition line.

At high chemical potential and low temperatures, a novel phase structure emerges: the chiral transition line splits into two branches. This behavior has previously been observed in a QM model RG study [55]; see left panel in Fig. 4 for our QM result. In accordance with the previous findings we find two critical points $(\mathrm{CPs})$ in this region: one on the outer transition branch at low $T$ and a further $\mathrm{CP}$ at higher temperature.

However, for the PQM model only one $\mathrm{CP}$ survives which is located on the inner branch of the transition line, at $\left(\mu_{\mathrm{CP}}, T_{\mathrm{CP}}\right)=(255,27) \mathrm{MeV}$, similar to the location for physical masses. Below this point, the transition is of first order, while the outer branch remains of second order. Note that due to the finite quark masses, the Polyakov-loop related transitions are still crossovers. Furthermore, the sharp transition in the chiral sector induces an additional peak in the temperature derivatives of the Polyakov loops. This interferes with the definition of the dark band in the PQM phase diagram and subsequently, the focusing of this band towards the critical point is not directly seen in this limit.

Furthermore, the chiral splitting is also reflected in the behavior of the sigma meson mass as a function of the chemical potential as illustrated in Fig. 5 for the PQM model at $T=30 \mathrm{MeV}$, i.e. just above the critical point. At low temperatures two minima, corresponding to the two branches of the chiral transition, appear in $m_{\sigma}(\mu)$. In contrast to the sigma mass, the pion mass remains zero until chiral symmetry is completely restored, which happens at the outer transition branch. The quark mass, on the other hand, is proportional to the chiral order parameter, $m_{q}=h \sigma$. Thus it shows a change in slope at the first transition and reaches zero at the second transition.

\section{Small pion mass}

Here we demonstrate how the splitting region in the phase diagram changes when the pion mass is increased towards the physical point.

The resulting phase structure for $m_{\pi}=50 \mathrm{MeV}$ is shown in Fig. 6. Due to the nonvanishing explicit chiral symmetry breaking, the chiral transition immediately becomes a crossover. In the low temperature/high chemical potential region we still observe a splitting in the chiral transition line and also the second minimum in the sigma meson mass persists. As the pion mass is further 

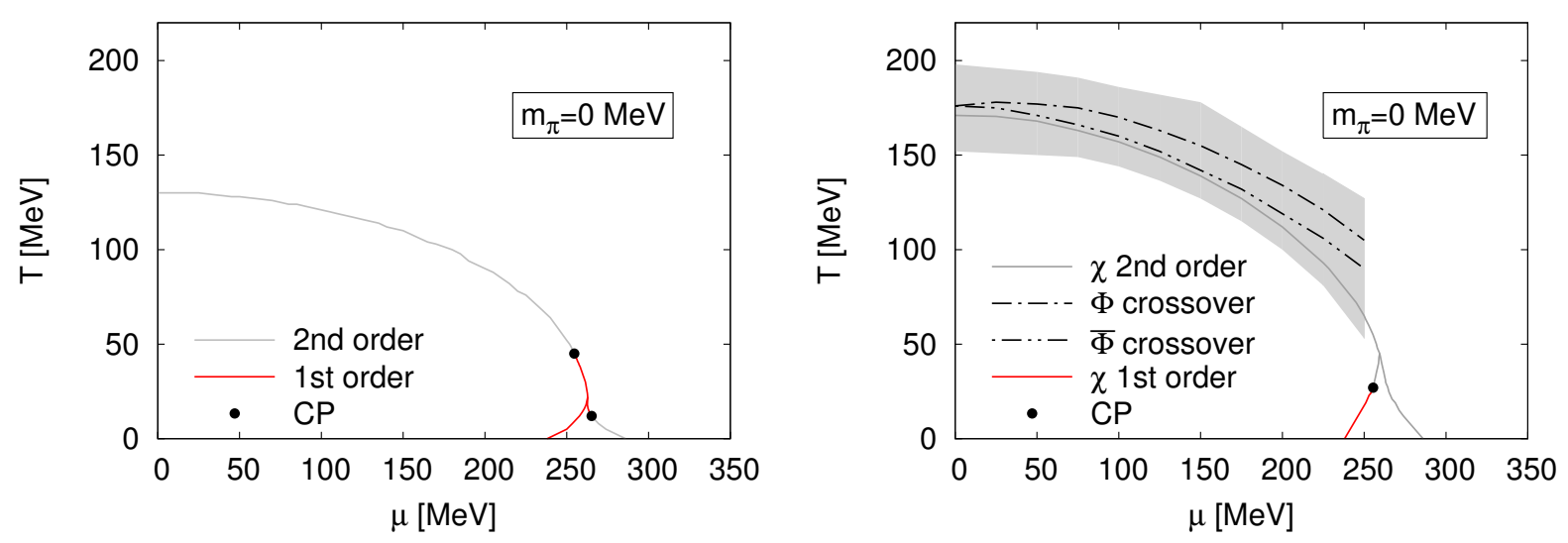

FIG. 4: Similar to the previous Fig. 3 but for the chiral limit; see text for more details.

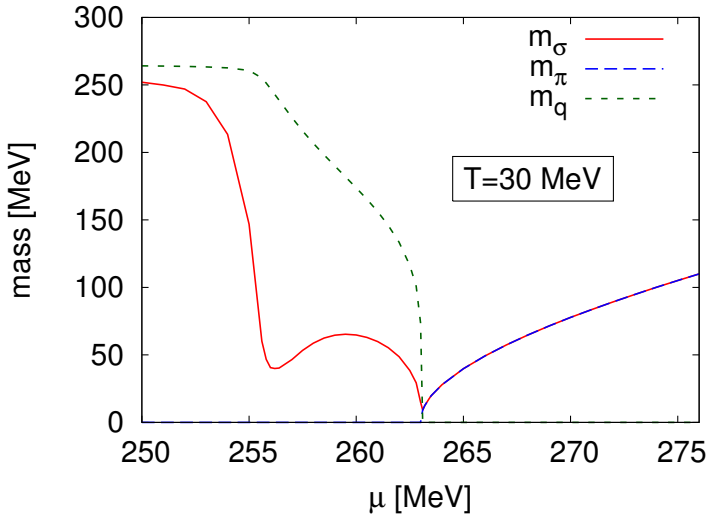

FIG. 5: Meson $\left(m_{\pi}, m_{\sigma}\right)$ and quark $\left(m_{q}\right)$ masses in the chiral limit as a function of $\mu$ for constant temperature $T=30 \mathrm{MeV}$. One extra minimum of $m_{\sigma}$ occur in the splitting region while $m_{\pi}$ vanishes until the second chiral transition is reached.

increased towards its physical value, however, the outer branch of the transition is weakened.

We also comment on the impact of the thermal and chemical potential modification of the initial conditions discussed in Sec. II B on the above effects: when the initial conditions of the flow are fixed in the vacuum and $T$-independent $T_{0}(\mu)$ is used, it is not possible to define a second branch of the chiral transition at the physical point. The inclusion of a $T$ and $\mu$ dependence of the initial action, $\Omega_{\Lambda}(T, \mu)$, as well as the more sophisticated definition of $T_{0}(T, \mu)$ in Eq. (8), however, enhances this effect once more, as shown in Fig. 7. In this figure we compare the $\mu$-derivative of the chiral order parameter for $T_{0}(\mu)$ and $\Omega_{\Lambda}(T, \mu)=\Omega_{\Lambda}(0,0)$ ("standard") to the result using $T_{0}(T, \mu)$ and $\Omega_{\Lambda}(T, \mu)$ ("enhanced") at physical masses. While both versions show a peak at $\mu_{\chi} \approx 293 \mathrm{MeV}$ - defining the (inner) transition branch the enhanced version shows a clear additional peak corresponding to the second branch. In fact, the line defined by this second peak agrees well with the one determined by Eq. (21) and is hence not explicitly shown in Fig. 3. A comparison of the phase structure in the standard and enhanced versions can be found in App. B.

Note also that the location of the critical point in temperature direction, $T_{\mathrm{CEP}}$, is only mildly sensitive to the pion mass. In all considered cases we find a critical point at $T_{\mathrm{CEP}} \approx 20-30 \mathrm{MeV}$. This is in contrast to previous PQM model results where $\left(T_{\mathrm{CEP}}, \mu_{\mathrm{CEP}}\right)$ depends strongly on $m_{\sigma}$ when $m_{\pi}, f_{\pi}$ and $m_{q}$ were fixed to their physical values; see [73] for mean-field results. When fluctuations beyond the mean-field approximation are taken into account, this observation still holds. Here, however, we vary only the explicit chiral symmetry parameter, which results in different values for e.g. $f_{\pi}$ and $m_{q}$.

On the other hand, the location of the critical point along the chemical potential axis, $\mu_{\mathrm{CEP}}$, is changed more drastically as $m_{\pi}$ is varied. This can be understood by noting that the critical chemical potential at vanishing temperature, $\mu_{c}(T=0)$, is related to the quark Fermi surface and hence to the quark mass which increases with $m_{\pi}$, and so does $\mu_{c}(T=0)$.

\section{THERMODYNAMICS}

In order to achieve deeper insights into the nature of the phase transitions and the impact of the Polyakov loop, we focus in the following on some thermodynamic observables.

\section{A. Thermodynamic observables}

The thermodynamic grand potential is obtained by evaluating the infrared effective average potential on the EoM (18)

$$
\Omega(T, \mu)=\left.\Omega_{k \rightarrow 0}(T, \mu)\right|_{\chi_{0}} .
$$



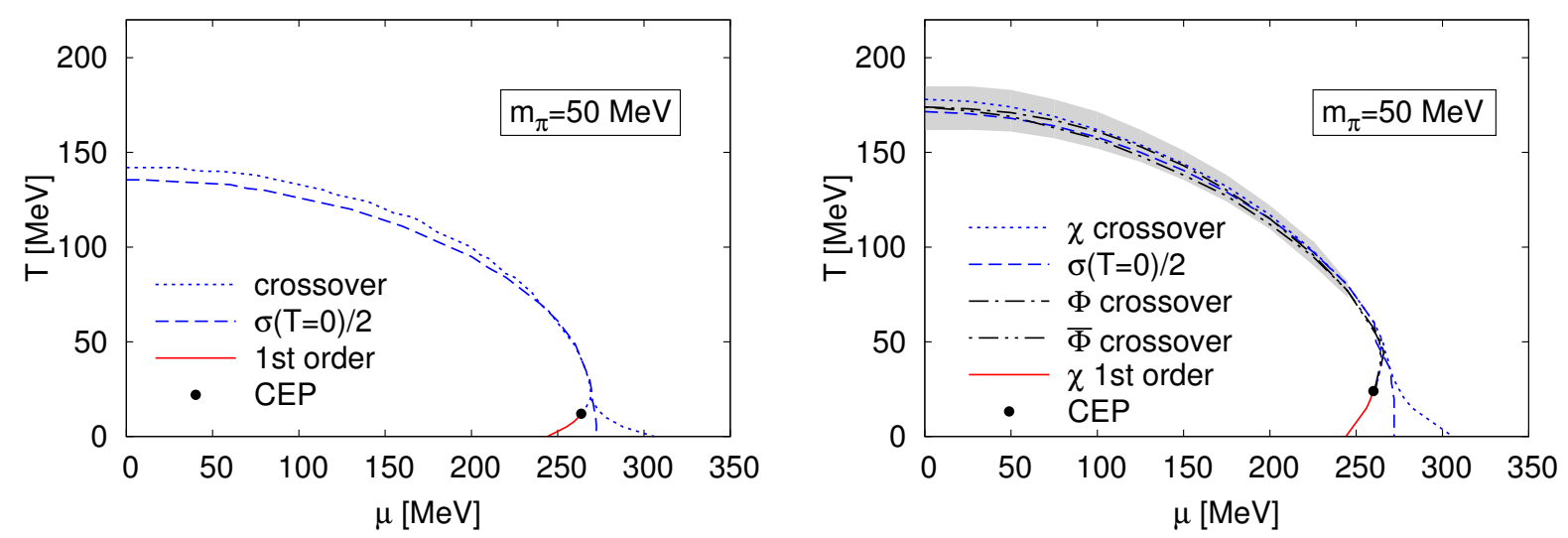

FIG. 6: Phase structure of the QM (left) and PQM (right) model for small pion masses $m_{\pi}=50 \mathrm{MeV}$. For these masses, the transition splitting persists, while it is washed out for increasing masses towards the physical point, cf. Fig. 3.

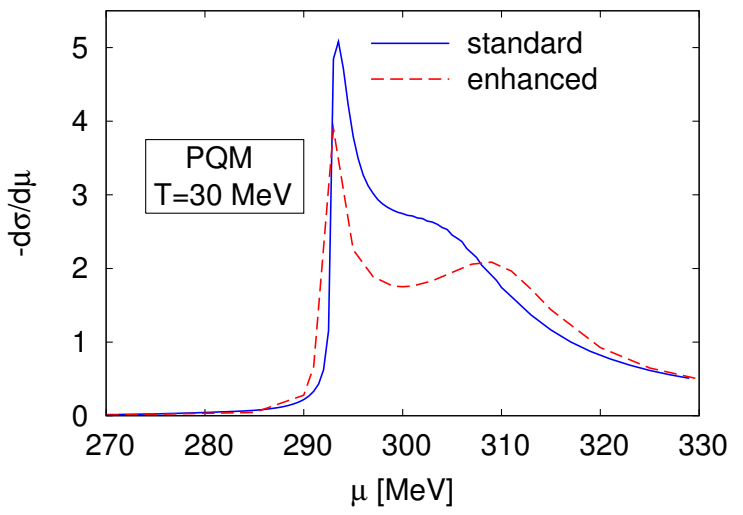

FIG. 7: Chemical potential derivative of the chiral order parameter vs $\mu$ at physical pion masses and low temperature. Including the $T$ and $\mu$ dependence of the UV potential, $\Omega_{\Lambda}(T, \mu)$ as well as the modified version of $T_{0}(\mu)$ a splitting in the chiral transition can be determined also at physical masses.

We begin with a discussion of the pressure which is defined as the negative value of the grand potential

$$
p(T, \mu)=-\Omega(T, \mu)+\Omega(0,0)
$$

and is normalized to zero in the vacuum. Since the pressure represents a thermodynamic potential, all further observables follow from this expression in the standard way by differentiation. For example, the first derivatives of the pressure with respect to $T$ and $\mu$ yield the entropy and quark number density

$$
s=\frac{\partial p(T, \mu)}{\partial T}, \quad n_{q}=\frac{\partial p(T, \mu)}{\partial \mu},
$$

respectively. For high temperatures and densities we use the Stefan-Boltzmann pressure of massless QCD, corresponding to an ideal gas of massless quarks and gluons for normalization. For $N_{f}$ flavors and $N_{c}$ colors this yields

$$
\begin{aligned}
\frac{p_{\mathrm{SB}}}{T^{4}} & =\frac{N_{f} N_{c}}{6}\left[\frac{7 \pi^{2}}{30}+\left(\frac{\mu}{T}\right)^{2}+\frac{1}{2 \pi^{2}}\left(\frac{\mu}{T}\right)^{4}\right] \\
& +\left(N_{c}^{2}-1\right) \frac{\pi^{2}}{45} .
\end{aligned}
$$

The expression in the first line denotes the fermionic contribution and the second line contains the gluonic part. Appropriate derivatives of this expression can be used to normalize other thermodynamic observables.

In addition we can define the energy density $\epsilon=-p+$ $T s+2 \mu n_{q}$ which is used to calculate the trace anomaly

$$
\frac{\Delta}{T^{4}}=\frac{\Theta_{\nu}^{\nu}}{T^{4}}=\frac{\epsilon-3 p}{T^{4}},
$$

which is related to the trace of the energy-momentum tensor $\Theta^{\mu \nu}$ and vanishes in a scale invariant theory. This quantity thus yields a measure for the breaking of conformal invariance of the system. Furthermore, this observable is also referred to as the interaction measure, since it quantifies the deviation from the equation of state of an ideal gas $\epsilon=3 p$, cf. Eq. (26).

\section{B. Mass sensitivity}

In the remaining section we investigate the thermodynamic observables, the impact of the Polyakov loop on these in hot and dense matter and study their mass sensitivity by varying the pion masses.

Figure 8 shows the pressure normalized by its StefanBoltzmann value for three fixed values of chemical potential and pion masses $m_{\pi}=138,50,0 \mathrm{MeV}$ from left to right. The largest chemical potential is chosen such that we pass close to the critical point. Results for the QM model are depicted in the upper panels, while the PQM calculation is presented in the bottom panels.

The $\mathrm{QM}$ pressure levels at $p / p_{\mathrm{SB}} \approx 0.6$, owing to the lack of gluonic degrees of freedom. At low temperatures, 

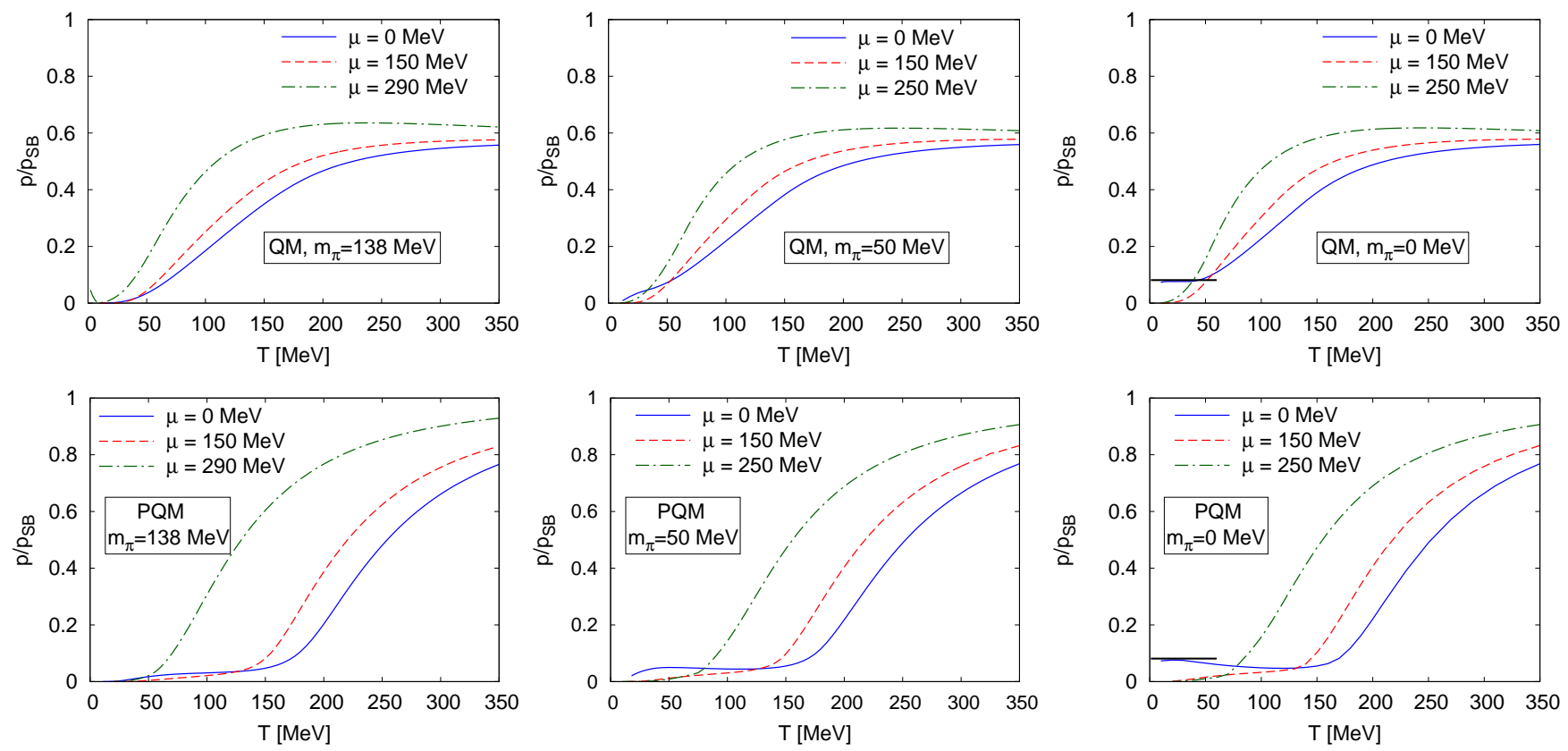

FIG. 8: Pressure normalized by the Stefan-Boltzmann pressure, Eq. (25), in the QM (upper panels) and PQM (lower panels) model, each for $m_{\pi}=138,50,0 \mathrm{MeV}$ (left to right).

the pressure is dominated by the lightest mesons, i.e. pions. Due to the complete lack of confinement in the QM truncation, the pressure rises almost immediately, while the slope becomes steeper for higher chemical potential. As the pion mass is lowered, the expected dominance of the Goldstone bosons in $p$ is visible [74]. In the chiral limit and at vanishing chemical potential, a plateau develops that is slightly below the expected value of $p / T^{4}=3 \pi^{2} / 90$ for a free gas of massless pions, denoted by the black line at low $T$.

Including the Polyakov loop, which represents a statistical implementation of confinement, the picture changes. Now, the pressure is almost constant in the hadronic phase. Again, a plateau develops at low temperatures that becomes more and more pronounced as we decrease $m_{\pi}$. With increasing temperature, as the quark masses decrease due to the restoration of chiral symmetry, the pressure rises more strongly. As the chemical potential increases, the chiral and deconfinement transition temperatures are lowered and the pressure rises strongly already at lower temperatures. It saturates at about $80 \%$ $90 \%$ of the Stefan-Boltzmann limit at high temperatures. A similar behavior is found for all pion masses.

When decreasing the pion mass, however, another problem arises: The coupling of the Polyakov-loop and quark sectors seem to be badly balanced. As a result, we find that the pressure increases slower than $T^{4}$ at $\mu=0$, resulting in a nonmonotonic ratio $p / p_{\mathrm{SB}}$. We emphasis that nevertheless, $p$ itself is a monotonically increasing function of temperature, as it should be.

Combining the energy density and the pressure as defined in Eq. (26), we obtain the interaction measure $\Delta / T^{4}$ which we show in Fig. 9. This quantity again illustrates nicely the improvement achieved by the inclusion of the Polyakov loop [44]. The QM result of this observable showed an unphysical two-peak structure at nonvanishing chemical potential. This effect is cured by the inclusion of gluonic degrees of freedom. The interaction measure is approximately zero at low temperatures and increases strongly around the phase transition. At high temperatures it decreases $\sim 1 / T^{2}$. Of course, the nonmonotonic behavior of $p / T^{4}$ at $m_{\pi}<138 \mathrm{MeV}$ also influences this quantity, which results in slightly negative scale anomaly at low $T$ for these masses. For physical masses, however, we find good agreement with recent lattice results at small $\mu[75,76]$.

\section{CONCLUSIONS AND OUTLOOK}

Based on a renormalization group analysis we have presented results on the phase structure and thermodynamics of the Polyakov-quark-meson truncation for two quark flavors, which serves as an effective model for lowenergy two-flavor QCD. Special emphasis is put on the influence of thermal and quantum fluctuations, that are crucial for a proper description of phase transitions. In particular, we have argued that the PQM model constitutes a well-controlled approximation to first-principles full QCD. One focus is given to the back-coupling of quarks to the glue sector of QCD which results in a $N_{f^{-}}$, $T$ - and $\mu$-dependent modification of the $T_{0}$ parameter in the Polyakov-loop potential.

At physical pion masses we find a chiral critical end- 

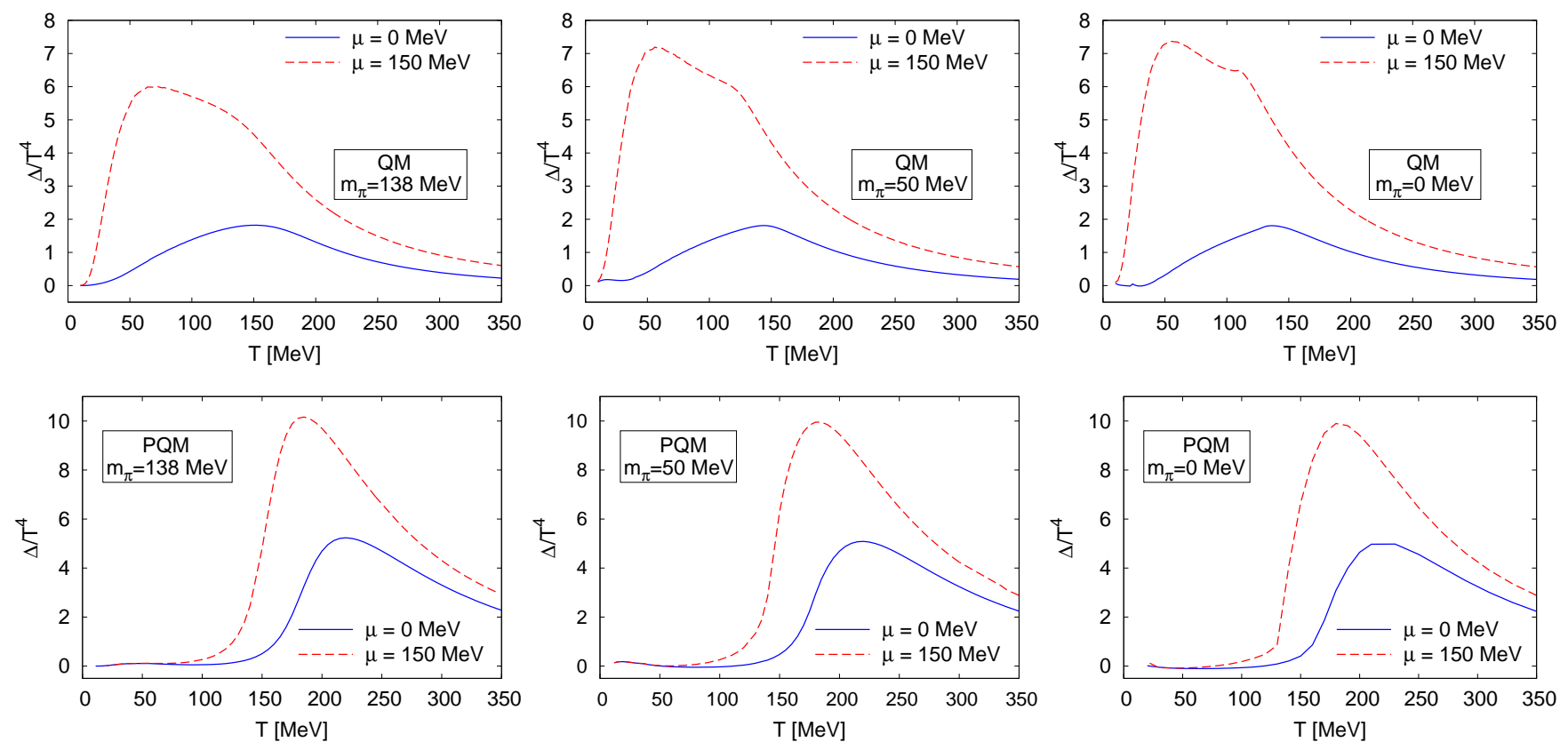

FIG. 9: Interaction measure $\Delta / T^{4}$ in the QM model (upper panels) and PQM model (lower panels), see text for details.

point at much lower temperatures than found in standard mean-field calculations. This nicely demonstrates the importance of fluctuations. Moreover, we can rule out the existence of a critical point for small chemical potential $\mu / T \approx 1-2$, see also [77] for corresponding lattice results.

When studying the mass sensitivity of the chiral phase diagram, we encounter an intriguing phase structure. In the chiral limit, at high chemical potential and low temperatures a splitting of the chiral transition line into two branches is observed. This splitting is not observed in the mean-field approximation in the same model, even if the vacuum term of the quark loop is taken into account. A similar effect has, however, previously been found in a FRG study of the quark-meson model [55]. Differences between the previous and the present work can be attributed to our higher value of the UV cutoff and different vacuum parameters. Interestingly, similar splitting effects also emerge in a finite volume FRG investigation within a two-flavor quark-meson model, cf. [78].

The splitting of the chiral transition is also reflected in a second minimum of the sigma meson mass as a function of the chemical potential as well as in the quark number susceptibility, which are sensitive to the chiral properties of the system.

Increasing the pion mass towards its physical value we observe that the splitting in the chiral transition line persists. The outer branch of the transition is weakened, which can for example be seen in the behavior of the sigma meson mass. At physical pion mass, the second transition is mostly washedout.

Of course, it would be interesting to study the vicinity of the critical point in more detail, but unfortunately some shortcomings of the Polyakov-loop potential pre- vent the computation of thermodynamic observables in the high chemical potential/low-temperature region. The calculation of the phase structure itself relies mainly on the EoM, i.e., on derivatives of the effective potential with respect to the order parameters. These are not affected by the above-mentioned problems, which still allows us to study the phase structure itself.

For a thorough study of the nature of the splitting region at small pion mass, the deficiency of the Polyakovloop potential poses serious problems. Regarding QCD, however, we are aware that in this high chemical potential region our present approximation is incomplete. At baryon chemical potential $\mu_{B}=3 \mu \sim 900 \mathrm{MeV}$, baryon degrees of freedom certainly play an important role in full QCD. Diquark fluctuations and baryonic effects are expected to have a sizeable impact, as also suggested by two-color results [67-70] and computations with isospin chemical potential, e.g. [79]. Thus, in order to produce reliable predictions of the physics in the vicinity of the QCD critical point - if it actually exists - an extension of the present model by the inclusion of these degrees of freedom is inevitable.

\section{Acknowledgements}

We thank J. Braun, L. Haas, L. Fister, M. Mitter, M. Puhr, J. Schaffner-Bielich, R. Stiele and M. Wagner for discussions and collaboration on related topics. This work is supported by the Helmholtz Alliance HA216/EMMI, by ERC-AdG-290623, by the FWF grant P24780-N27 and by CompStar, a research networking programme of the European Science Foundation. 
TKH was supported by a DOC-fFORTE-fellowship of the ÖAW and by the FWF through DK-W1203-N16.

\section{Appendix A: Differential Evolution Algorithm}

Usually, finding the roots of a higher-dimensional system of nonlinear equations, such as the EoM, Eq. (18), a natural choice would be Newton's method. This method converges quadratically if the initial values are already close to the final solution. Applied to our RG calculation a huge number of iteration steps, of the order $\mathcal{O}\left(10^{4}\right)$, are actually needed to gain an acceptable numerical precision of the solution. However, each of these steps involves a complete RG evolution which increases the CPU time drastically. Hence, for the computation of thermodynamic quantities and higher derivatives, in particular for low temperatures, an alternative method that requires less RG evolutions is inevitable. Such an alternative technique is provided by the differential evolution (DE) algorithm for global optimization [80] which enables the solution of our three-dimensional system of the nonlinear EoM, Eq. (18), to high numerical precision with fewer RG evolutions.

In detail the system Eq. (18) is solved as follows: we discretize the effective potential on a one dimensional grid (in radial direction $\sigma$ ) and determine for arbitrary fixed initial values of the remaining variables $(\Phi, \bar{\Phi})$ the potential minimum after the $\mathrm{RG}$ evolution. Then the EoM in $\sigma$ direction is fulfilled and we are left with a twodimensional subsystem for the remaining Polyakov-loop variables.

For these variables we define the cost function

$$
f(\Phi, \bar{\Phi})=\left(\frac{\partial \Omega_{k \rightarrow 0}}{\partial \Phi}\right)^{2}+\left(\frac{\partial \Omega_{k \rightarrow 0}}{\partial \bar{\Phi}}\right)^{2}
$$

and apply the DE algorithm which we describe in the following. 12.38.Aw 11.10.-z 11.30.Rd 12.38.-t In the first step of the DE algorithm, an array $x_{i j}$ of $i=1, \ldots, N$ pairs $\left(\Phi_{i}, \bar{\Phi}_{i}\right)$, called target vector, is generated by randomly choosing values in a user-specified initial interval. For the present application we use $\left(\Phi_{i}, \bar{\Phi}_{i}\right) \in[0,1] \times[0,1]$ and typically the target vector has a length of $N=$ $10-20$.

Step two consists of defining the trial vector $v_{i j}$, which is obtained from $x_{i j}$ by a randomization procedure. For the details of this procedure, we refer the reader to [80].

Subsequently, the cost function is evaluated on the newly defined array $v_{i j}$ and compared to its value on the current target vector $x_{i j}$. Note that this is the only step that involves the RG evolution. If, for a given index $i$, the result of the trial vector is smaller than the one of the target vector, the corresponding entry in the target vector is replaced by the one from the trial vector. The overall minimum of the cost function on the modified target vector can then easily be calculated and compared to the user-specified criterion, e.g. $f\left(\Phi_{\min }, \bar{\Phi}_{\min }\right) \leq 10^{-3}$. If

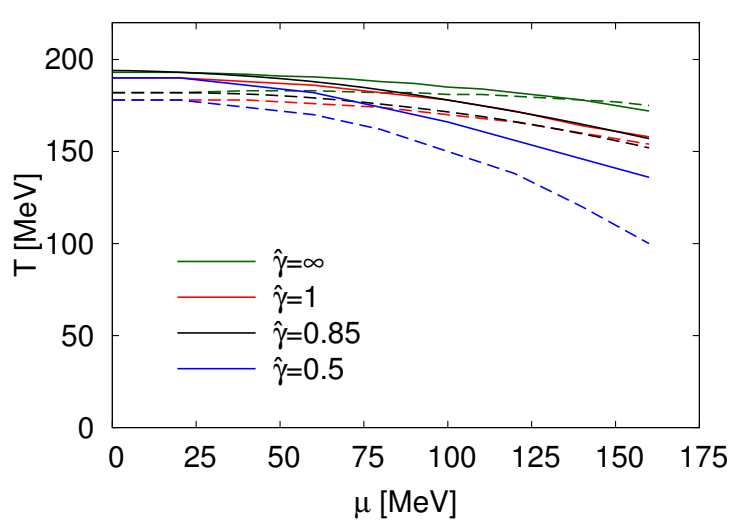

FIG. 10: Dependence of the phase structure on our choice of the parameter $\hat{\gamma}$ in $T_{0}\left(N_{f}, T, \mu\right)$. The solid lines denote the chiral transition while the dashed lines correspond to the transition related to the Polyakov-loop, $\Phi$. See text for a detailed discussion.

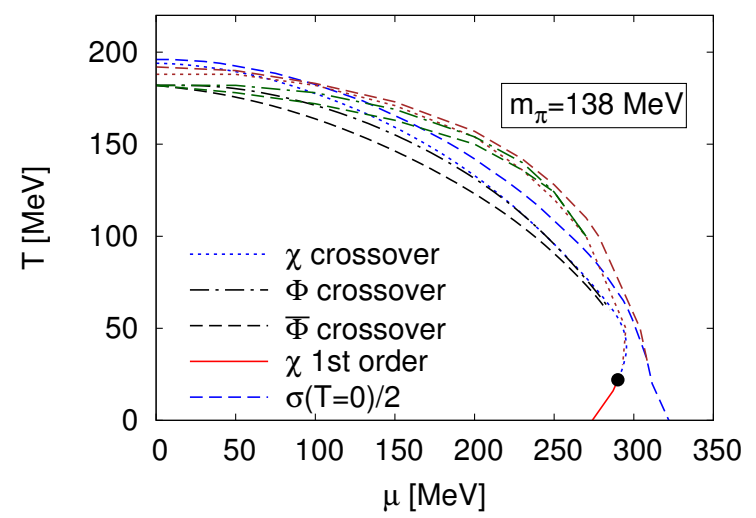

FIG. 11: Phase structure at physical masses with (upper lines) and without (lower lines) modified UV potential and $\theta_{b}$.

this criterion is not fulfilled yet, the procedure is repeated from step two.

With this algorithm it is possible to solve the EoM with an accuracy of at least $\mathcal{O}\left(10^{-3}\right)$ after $\mathcal{O}\left(10^{2}\right)$ generations, which corresponds to $\mathcal{O}\left(10^{3}\right) \mathrm{RG}$ evolutions. For comparison, the same number of RG evolutions using Newton's method would typically yield roots of the order of $\mathcal{O}\left(10^{2}\right)$ only.

\section{Appendix B: Parameter Dependence of the Phase Structure}

In this appendix we briefly comment on the sensitivity of the phase structure on our choice of parameters for the physical mass point.

First, we consider the parameter $\hat{\gamma}$ in Eq. (9). This quantity measures the strength of the chemical potential dependence of $T_{0}$. In Fig. 10 we show the chiral 
(solid line) and Polyakov-loop related (dashed line) transitions at low and intermediate chemical potentials for $\hat{\gamma}=0.5,0.85,1, \infty$ (blue, black, red and green lines, respectively). For better readability we have omitted the line related to the conjugate Polyakov-loop, $\bar{\Phi}$. As can be seen in Figs. 3, 4 and 6, the corresponding transition always lies below the $\Phi$-related transition line and both agree within their width. Furthermore, the curvature of both deconfinement-related transitions is rather similar.

Fig. 10 shows that the curvature of the deconfinement transition increases continuously as $\hat{\gamma}$ is lowered from infinity, which corresponds to constant $T_{0}$. The full phase diagramm in this case can be found in Ref. [3]. The curvature of the chiral transition increases as well, but the effect is much weaker. In the present work we have used $\hat{\gamma}=0.85$, which is the value that is suggested by a comparison to the HDL approximation; see Fig. 3 for the full phase structure. For smaller values, the curvature increases even more, see e.g. the blue curve in Fig. 10, corresponding to $\hat{\gamma}=0.5$. Such values are unrealistic, since they correspond to a $T_{0}(\mu)$ that approaches zero already at low chemical potentials.
Next, we investigate the influence of the temperature and chemical potential dependent initial condition, $\Omega_{\Lambda}(T, \mu)$, as well as the temperature dependence of $T_{0}$, Eq. (10), on the phase structure. It was already shown in Fig. 7 that the splitting of the chiral transition at high chemical potential becomes stronger at physical masses when this enhancement is taken into account ("enhanced version"). In Fig. 11 we compare the phase structure for the standard and enhanced versions. The phase structure with temperature independent $T_{0}(\mu)$ and fixed initial values $\Omega_{\Lambda}(T, \mu)=\Omega_{\Lambda}(0,0)$ (lower, blue and black lines) is compared to the enhanced version (upper, brown and green lines). The results coincide at small chemical potentials. The curvature of both transitions at low $\mu$ is smaller in the enhanced version, yielding better agreement with the lattice results. At higher chemical potentials, the curvature increases once more, bending towards the result obtained in the standard version at low T. As already pointed out in the discussion of Fig. 7, in the enhanced version the chiral transition line defined by Eq. (21) agrees with the outer branch of the chiral transition in this region.
[1] J. Braun, L. M. Haas, F. Marhauser, and J. M. Pawlowski, Phys.Rev.Lett. 106, 022002 (2011), 0908.0008.

[2] V. Skokov, B. Stokic, B. Friman, and K. Redlich, Phys. Rev. C82, 015206 (2010), 1004.2665.

[3] T. K. Herbst, J. M. Pawlowski, and B.-J. Schaefer, Phys.Lett. B696, 58 (2011), 1008.0081.

[4] J. M. Pawlowski, AIP Conf.Proc. 1343, 75 (2011), 1012.5075 .

[5] J. Braun and A. Janot, Phys.Rev. D84, 114022 (2011), 1102.4841

[6] J. Braun and T. K. Herbst (2012), 1205.0779.

[7] C. S. Fischer and J. Luecker, Phys.Lett. B718, 1036 (2013), 1206.5191.

[8] D. F. Litim and J. M. Pawlowski (1998), hep-th/9901063.

[9] J. Berges, N. Tetradis, and C. Wetterich, Phys.Rept. 363, 223 (2002), hep-ph/0005122.

[10] J. Polonyi, Central Eur.J.Phys. 1, 1 (2003), hepth/0110026.

[11] J. M. Pawlowski, Annals Phys. 322, 2831 (2007), hepth/0512261.

[12] H. Gies, Lect.Notes Phys. 852, 287 (2012), hep$\mathrm{ph} / 0611146$.

[13] B.-J. Schaefer and J. Wambach, Phys.Part.Nucl. 39, 1025 (2008), hep-ph/0611191.

[14] B.-J. Schaefer, Phys.Atom.Nucl. 75 (2012), 1102.2772.

[15] J. Braun, J.Phys.G G39, 033001 (2012), 1108.4449.

[16] L. von Smekal, Nucl.Phys.Proc.Suppl. 228, 179 (2012), 1205.4205

[17] R. Alkofer and L. von Smekal, Phys. Rept. 353, 281 (2001), hep-ph/0007355.

[18] C. D. Roberts and S. M. Schmidt, Prog. Part. Nucl. Phys. 45, S1 (2000), nucl-th/0005064.

[19] C. S. Fischer, J.Phys.G G32, R253 (2006), hep$\mathrm{ph} / 0605173$.
[20] C. S. Fischer, A. Maas, and J. M. Pawlowski, Annals Phys. 324, 2408 (2009), 0810.1987.

[21] D. Binosi and J. Papavassiliou, Phys.Rept. 479, 1 (2009), 0909.2536 .

[22] A. Maas, Phys.Rept. 524, 203 (2013), 1106.3942.

[23] F. Karsch, Lect. Notes Phys. 583, 209 (2002), heplat/0106019.

[24] O. Philipsen, Eur. Phys. J. ST 152, 29 (2007), 0708.1293.

[25] P. de Forcrand, PoS LAT2009, 010 (2009), 1005.0539.

[26] P. Cea, L. Cosmai, M. D'Elia, C. Manneschi, and A. Papa, Phys. Rev. D80, 034501 (2009), 0905.1292.

[27] S. Ejiri et al. (WHOT-QCD Collaboration), Central Eur.J.Phys. 10, 1322 (2012), 1203.3793.

[28] G. Aarts, Phys. Rev. Lett. 102, 131601 (2009), 0810.2089 .

[29] C. Schmidt, PoS LAT2006, 021 (2006), heplat/0610116.

[30] E. Seiler, D. Sexty, and I.-O. Stamatescu (2012), 1211.3709.

[31] G. Aarts, F. A. James, J. M. Pawlowski, E. Seiler, D. Sexty, et al., JHEP 1303, 073 (2013), 1212.5231.

[32] K. Fukushima and C. Sasaki (2013), 1301.6377.

[33] E. Megias, E. Ruiz Arriola, and L. Salcedo, Phys.Rev. D74, 065005 (2006), hep-ph/0412308.

[34] K. Fukushima, Phys.Lett. B591, 277 (2004), hep$\mathrm{ph} / 0310121$.

[35] B.-J. Schaefer, J. M. Pawlowski, and J. Wambach, Phys.Rev. D76, 074023 (2007), 0704.3234.

[36] P. N. Meisinger and M. C. Ogilvie, Phys.Lett. B379, 163 (1996), hep-lat/9512011.

[37] R. D. Pisarski, Phys.Rev. D62, 111501 (2000), hep$\mathrm{ph} / 0006205$.

[38] A. Mocsy, F. Sannino, and K. Tuominen, Phys.Rev.Lett. 92, 182302 (2004), hep-ph/0308135.

[39] A. Dumitru, Y. Hatta, J. Lenaghan, K. Orginos, and 
R. D. Pisarski, Phys.Rev. D70, 034511 (2004), hepth/0311223.

[40] C. Ratti, M. A. Thaler, and W. Weise, Phys.Rev. D73, 014019 (2006), hep-ph/0506234.

[41] S. K. Ghosh, T. K. Mukherjee, M. G. Mustafa, and R. Ray, Phys.Rev. D73, 114007 (2006), hep-ph/0603050.

[42] C. Sasaki, B. Friman, and K. Redlich, Phys. Rev. D75, 074013 (2007), hep-ph/0611147.

[43] Y. Sakai, K. Kashiwa, H. Kouno, and M. Yahiro, Phys.Rev. D77, 051901 (2008), 0801.0034.

[44] B.-J. Schaefer, M. Wagner, and J. Wambach, Phys.Rev. D81, 074013 (2010), 0910.5628.

[45] B. W. Mintz, R. Stiele, R. O. Ramos, and J. SchaffnerBielich, Phys. Rev. D 87, 036004 (2013), 1212.1184.

[46] L. M. Haas, J. Braun, and J. M. Pawlowski, AIP Conf.Proc. 1343, 459 (2011), 1012.4735.

[47] F. Marhauser and J. M. Pawlowski (2008), 0812.1144.

[48] K.-I. Kondo, Phys. Rev. D82, 065024 (2010), 1005.0314.

[49] T. K. Herbst, J. M. Pawlowski, and B.-J. Schaefer, Acta Phys.Polon.Supp. 5, 733 (2012), 1202.0758.

[50] J. Braun, Eur. Phys. J. C64, 459 (2009), 0810.1727.

[51] J. Braun, H. Gies, and J. M. Pawlowski, Phys.Lett. B684, 262 (2010), 0708.2413.

[52] J. Braun, A. Eichhorn, H. Gies, and J. M. Pawlowski, Eur.Phys.J. C70, 689 (2010), 1007.2619.

[53] L. Fister and J. M. Pawlowski (2013), 1301.4163.

[54] L. M. Haas, R. Stiele, J. Braun, J. M. Pawlowski, and J. Schaffner-Bielich, Phys.Rev. D87, 076004 (2013), 1302.1993.

[55] B.-J. Schaefer and J. Wambach, Nucl.Phys. A757, 479 (2005), nucl-th/0403039.

[56] B.-J. Schaefer and J. Wambach, Phys.Rev. D75, 085015 (2007), hep-ph/0603256.

[57] C. Wetterich, Phys.Lett. B301, 90 (1993).

[58] J. Braun, K. Schwenzer, and H.-J. Pirner, Phys.Rev. D70, 085016 (2004), hep-ph/0312277.

[59] J. Beringer (Particle Data Group) et al., Phys. Rev. D86, 010001 (2012).

[60] J. Braun, L. Fister, L. M. Haas, and J. M. Pawlowski, in preparation (2013).

[61] E. Nakano, B.-J. Schaefer, B. Stokic, B. Friman, and
K. Redlich, Phys.Lett. B682, 401 (2010), 0907.1344.

[62] V. Skokov, B. Friman, E. Nakano, K. Redlich, and B.-J. Schaefer, Phys.Rev. D82, 034029 (2010), 1005.3166.

[63] B.-J. Schaefer and M. Wagner, Phys.Rev. D85, 034027 (2012), 1111.6871.

[64] Y. Aoki, G. Endrodi, Z. Fodor, S. Katz, and K. Szabo, Nature 443, 675 (2006), hep-lat/0611014.

[65] L. McLerran and R. D. Pisarski, Nucl. Phys. A 796 (2007), arXiv:0706.2191 [hep-ph]].

[66] C. S. Fischer, J. Luecker, and J. A. Mueller, Phys.Lett. B702, 438 (2011), 1104.1564.

[67] C. Ratti and W. Weise, Phys.Rev. D70, 054013 (2004), hep-ph/0406159.

[68] S. Hands, S. Kim, and J.-I. Skullerud, Eur. Phys. J. C48, 193 (2006), hep-lat/0604004.

[69] T. Brauner, K. Fukushima, and Y. Hidaka, Phys.Rev. D80, 074035 (2009), 0907.4905.

[70] N. Strodthoff, B.-J. Schaefer, and L. von Smekal, Phys.Rev. D85, 074007 (2012), 1112.5401.

[71] B.-J. Schaefer and M. Wagner, Central Eur.J.Phys. 10, 1326 (2012), 1203.1883.

[72] R. D. Pisarski and F. Wilczek, Phys.Rev. D29, 338 (1984).

[73] B.-J. Schaefer and M. Wagner, Phys.Rev. D79, 014018 (2009), 0808.1491.

[74] B.-J. Schaefer and H.-J. Pirner, Nucl.Phys. A660, 439 (1999), nucl-th/9903003.

[75] S. Borsanyi, S. Durr, Z. Fodor, C. Hoelbling, S. D. Katz, et al., JHEP 1208, 126 (2012), 1205.0440.

[76] A. Bazavov (HotQCD Collaboration), Nucl.Phys.A (2012), 1210.6312

[77] G. Endrodi, Z. Fodor, S. Katz, and K. Szabo, JHEP 1104, 001 (2011), 1102.1356.

[78] J. Braun, B. Klein, B.-J. Schaefer, and R.-A. Tripolt, in preparation (2013).

[79] K. Kamikado, N. Strodthoff, L. von Smekal, and J. Wambach, Phys.Lett. B718, 1044 (2013), 1207.0400.

[80] R. Storn and K. Price, Journal of Global Optimization 11, 341 (1997). 